\title{
Greig cephalopolysyndactyly syndrome
}

INSERM

\section{Source}

INSERM. (1999). Orphanet: an online rare disease and orphan drug data base. Greig cephalopolysyndactyly syndrome. ORPHA:380

Greig cephalopolysyndactyly syndrome (GCPS) is a pleiotropic, multiple congenital anomaly syndrome. 